\title{
Expression of S100A4 combined with reduced E-cadherin expression predicts patient outcome in malignant melanoma
}

\author{
Kristin Andersen ${ }^{1}$, Jahn M Nesland ${ }^{2}$, Ruth Holm², Vivi A Flørenes ${ }^{2}$, Øystein Fodstad ${ }^{1}$ \\ and Gunhild M Mælandsmo ${ }^{1}$ \\ ${ }^{1}$ Department of Tumor Biology, Institute of Cancer Research and ${ }^{2}$ Department of Pathology, The Norwegian \\ Radium Hospital, University of Oslo, Montebello, Norway
}

\begin{abstract}
The aim of the present study was to analyze the expression of S100A4 and E-cadherin in a panel of primary and metastatic malignant melanoma, and to correlate the expression level to clinicopathological parameters. The expression of S100A4 was examined by immunohistochemistry in 99 superficial spreading and 60 nodular primary melanomas, while the expression of E-cadherin was analyzed in 92 superficial spreading and 52 nodular lesions from the same panel. The expression levels of S100A4 and E-cadherin in the biopsies were inversely correlated, with S100A4 being expressed at the highest frequency in the nodular and E-cadherin in the superficial spreading lesions, respectively. When analyzing the melanoma subgroups separately, it was revealed that expression of S100A4 had a more significant impact on patient outcome in early superficial spreading melanomas than in the nodular subtype, while E-cadherin expression did not predict patient outcome in any of the subgroups. When examining all the patients, both markers give clinical information as predictors for disease-free survival, but when combining the expression of the two markers, a stronger significant correlation between high E-cadherin expressing/S100A4 negative biopsies and increased disease-free survival $(P=0.002)$ was revealed, demonstrating the importance of examining the expression of more than one factor involved in the metastatic cascade when predicting patient outcome. We have also evaluated the relationship between the expression of these two antigens and cell cycle and signal transduction factors.
\end{abstract}

Modern Pathology (2004) 17, 990-997, advance online publication, 30 April 2004; doi:10.1038/modpathol.3800151

Keywords: prognostic factor; early melanoma; metastasize; cell-cell adhesion

The incidence of malignant melanomas is increasing, and once metastasized the prognosis is poor. Thus, early identification of patients with a high risk of progression is of major importance. Besides thickness of the primary tumor, few prognostic markers predicting patient outcome are available. The serum level of S100B, an S100 family member, has been demonstrated to be a valuable and reliable indicator for clinical progression and response to therapy in several studies. ${ }^{1}$ A major disadvantage of $\mathrm{S} 100 \mathrm{~B}$ is, however, the poor prognostic impact in early-stage melanoma patients. ${ }^{2}$

The S100 family of $\mathrm{Ca}^{2+}$ binding proteins comprises 21 members, each exhibiting a unique expression pattern in human tissues, and exerting

Correspondence: GM Mælandsmo, PhD, Department of Tumor Biology, The Norwegian Radium Hospital, Montebello, N-0310 Oslo, Norway.

E-mail: g.m.malandsmo@labmed.uio.no

Received 5 January 2004; accepted 5 March 2004; published online 30 April 2004 different functions. ${ }^{3}$ Another member of the family, the S100A4 protein, has previously been associated with increased metastatic capacity of cancer cells, ${ }^{4,5}$ but how S100A4 exerts its putative metastasispromoting effects is largely unknown. Previously, we found an association between the expression of S100A4, matrix metalloproteinase (MMP) activity and the metastatic capacity of human osteosarcoma cells. ${ }^{6}$ Other studies have suggested a role in cell motility, ${ }^{7,8}$ and Ambartsumian et $a l^{9}$ reported that S100A4 secreted from tumor cells could cause cancer progression through stimulation of angiogenesis. Recently, a number of studies have suggested a correlation between the expression of S100A4 and clinical outcome in various tumor types (gall bladder, esophageal and colon). ${ }^{10-12}$ Of special interest, S100A4 was found to be the most significant predictor of overall survival, in stage I and II breast carcinoma patients, and also when welldocumented clinical and pathological parameters such as lymph node infiltration and estrogen receptor status were taken into consideration. ${ }^{13} \mathrm{In}$ 
other studies, an inverse association between S100A4 and E-cadherin has been revealed. ${ }^{14-16}$ Ecadherin mediates homophilic calcium-dependent cell-cell adhesion, ${ }^{17,18}$ and is the prime mediator of melanocyte adhesion to keratinocytes in vitro. ${ }^{19}$ The loss of E-cadherin expression occurs in a variety of human tumors and is assumed to be an important step in the progression from a tumor in situ to an invasive and metastasizing lesion. ${ }^{20,21}$

The objective of the present study was to investigate by immunohistochemistry the expression pattern of S100A4 and E-cadherin in a panel of superficial spreading and nodular malignant melanomas, and to evaluate whether these two proteins alone, or in combination, might have any impact as prognostic makers in this type of cancer. In addition, we evaluated the relationship between the expression of S100A4 and E-cadherin and cell cycle and signal transduction factors previously analyzed in this panel.

For the first time, we report here that the expression of S100A4 is related to clinical outcome also in malignant melanoma, and demonstrate that expression of S100A4 combined with reduced expression of E-cadherin might be useful predictors of patient outcome in this type of cancer.

\section{Methods}

\section{Specimens}

Formalin-fixed, paraffin-embedded tumor tissue from 159 primary malignant melanomas, 62 metastases and eight benign nevi was randomly collected from the archives of The Norwegian Radium Hospital and regional hospitals and analyzed for expression of S100A4. Both primary (13 originating from superficial spreading and 10 from nodular lesions) and metastatic material was collected from 23 patients. Of the primary tumors, an experienced pathologist classified 99 as superficial spreading and 60 as nodular tumors. The lesions classified as nodular melanomas showed no sign of radial tumor cell growth in the epidermis. The median depth of the superficial spreading and nodular tumors was $1.8 \mathrm{~mm}$ (range $0.11-12.00 \mathrm{~mm}$ ) and $4.52 \mathrm{~mm}$ (range 0.95-15.00 mm), respectively. The median age of the whole group of patients was 53.3 years (range 19-97 years), and the median follow-up of patients still alive $(n=125)$ was 142.4 months (range 26-361 months). Of the metastatic lesions, 30 and 32 were developed from superficial spreading and nodular melanomas, respectively. From this panel, 144 primary malignant melanomas and 53 metastases were randomly selected for analysis of E-cadherin expression. Of the primary tumors, 92 were classified as superficial spreading and 52 as nodular tumors. Of the metastatic lesions, 26 and 27 were developed from superficial spreading and nodular melanomas, respectively. Treatment of the patients was according to WHO standard.

\section{Immunohistochemical Staining}

Sections from formalin-fixed, paraffin-embedded tissue were immunostained using the biotin-streptavidin-peroxidase method (Supersensitive Immunodetection System, LP000-UL; Biogenex, San Ramon, CA, USA) and the Optimax Plus Automated Cell Staining System (Biogenex). Deparaffinized sections were microwaved in $1 \mathrm{mM}$ EDTA ( $\mathrm{pH}$ 8.0) for $2 \times 5 \mathrm{~min}$ at $800 \mathrm{~W}$ to unmask epitopes. After treatment with $1 \%$ hydrogen peroxidase for $10 \mathrm{~min}$ to block endogenous peroxidase, the sections were incubated with rabbit polyclonal anti-S100A4 (DAKO, Glostrup, Denmark), diluted 1:300, for $30 \mathrm{~min}$ at room temperature, or with monoclonal mouse anti-E-cadherin (HECD-1) (Zymed ${ }^{\mathbb{R}}$ Laboratories Inc., San Francisco, CA, USA), diluted 1:1000, overnight at room temperature. The sections were thereafter incubated with biotin-labeled secondary antibody (1:30) and streptavidin-peroxidase (1:30) for $20 \mathrm{~min}$ each. Tissue was stained for $5 \mathrm{~min}$ with $0.05 \% 3,3^{\prime}$-diaminobenzidine tetrahydrochloride freshly prepared in $0.05 \mathrm{M}$ Tris-HCl buffer ( $\mathrm{pH} 7.6$ ) containing $0.024 \%$ hydrogen peroxidase and then counterstained with hematoxylin, dehydrated, and mounted in Diatex. All dilutions were made with phosphate-buffered saline, $\mathrm{pH} 7.4$, containing $1 \%$ bovine serum albumin. The specificity of the S100A4 antibody is described elsewhere. ${ }^{22,23}$ Positive and negative controls were included, the latter obtained by replacement of the primary antibody with normal polyclonal rabbit IgG of the same subclass and concentration, and in addition, incubation of sections with rabbit polyclonal antiS100A4 preabsorbed with $100 \mu \mathrm{g} / \mathrm{ml}$ recombinant S100A4. As a negative control for E-cadherin staining, the primary antibody was replaced with mouse myeloma protein of the same subclass and concentration as the primary antibody. One experienced pathologist blindly evaluated each panel of samples, and semiquantitatively estimated the number of stained tumor cells using four categories: $(-)$, none; $(+),<5 \% ;(++), 5-49 \%$; and $(+++)$, 50-100\%. For S100A4, cytoplasmic and nuclear staining was recorded as individual variables, while cytoplasmic and membranous staining was scored as individual variables for E-cadherin.

\section{Statistical Analysis}

The relationship between tumor thickness and the expression of S100A4 or E-cadherin was evaluated nonparametrically using the Mann-Whitney test, while comparisons between S100A4, E-cadherin and other proteins were performed using Pearsons $\chi^{2}$ test. The Kaplan-Meier survival estimates and log-rank tests were used to evaluate survival data. A value of $P<0.05$ was considered as statistically significant. All statistical evaluations were performed according to the SPSS statistical package (SPSS Inc., Chicago, IL, USA). 


\section{Results}

\section{S100A4 and E-cadherin Expression Levels in Primary and Metastatic Melanomas}

Formalin-fixed paraffin-embedded tissue from 159 primary (99 superficial spreading and 60 nodular) and 62 metastatic (32 superficial spreading and 30 nodular) melanomas was analyzed by immunohistochemistry for expression of the S100A4 protein. In the primary lesions, cytoplasmic expression of S100A4 was observed in $44 \%$ of the superficial spreading tumors, while $77 \%$ of the nodular melanomas showed S100A4 staining (Figure 1a and Table 1a). Of the biopsies expressing S100A4 in the cytoplasm, $42 \%$ of the nodular as compared to only $14 \%$ of the superficial spreading melanomas demonstrated positive staining in more than $50 \%$ of the cells. Nuclear S100A4 was also observed more often in the nodular (42\%) than in the superficial spreading (22\%) subgroup of primary melanomas. In contrast, when recording the number of metastatic biopsies expressing S100A4 in the cytoplasm, the difference seen between nodular and superficial spreading lesions was obliterated.

Of the metastatic tumors originating from nodular or superficial spreading lesions, 40 and $47 \%$ respectively, demonstrated cytoplasmic S100A4 staining. In the metastases from nodular lesions, only $13 \%$ showed a high degree of S100A4 staining compared to $34 \%$ in the superficial spreading subgroup, suggesting that in the nodular melanomas the high expression seen in the primary lesions is lost during tumor progression. In 23 cases, primary and metastatic tumor samples from the same patient could be examined. In contrast to what was observed when we examined the whole panel of nodular melanomas, no clear trends in the distribution of S100A4 expression between the primary and the corresponding metastatic lesions was detected in this small panel consisting of only 10 cases. Eight melanocytic nevi were included in the analysis, and none of these was found to express S100A4 in the cytoplasm, while nuclear staining was detected in six of the cases (Table 1a).

The majority of the samples, 144 primary (92 superficial spreading and 52 nodular) and 53 metastatic (26 superficial spreading and 27 nodular) lesions, as well as the nevi, were also analyzed for protein expression of E-cadherin. All the nevi were found to have membranous E-cadherin, while seven expressed the protein in the cytoplasm (Table 1b). A high percentage of the lesions, $87 \%$ of the superficial spreading and $75 \%$ of the nodular, expressed E-cadherin in the cytoplasm. Notably, $64 \%$ of the superficial spreading compared to only $29 \%$ of the nodular showed positive staining in more than $50 \%$ of the cells, and in the superficial spreading subgroup both membranous and cytoplasmic staining were reduced in the metastases compared to the primary lesions. Altogether, these observations suggest that E-cadherin expression is lost or reduced in the more advanced tumors.

\section{Expression of S100A4 and E-cadherin in Relation to Clinical Parameters}

The expression of S100A4 and E-cadherin was examined in relation to clinical parameters of melanoma progression. In a previous report, internalization of E-cadherin and relocation to other parts of the cell membrane as a fully functional adhesion molecule has been observed. ${ }^{24}$ In the present study, an
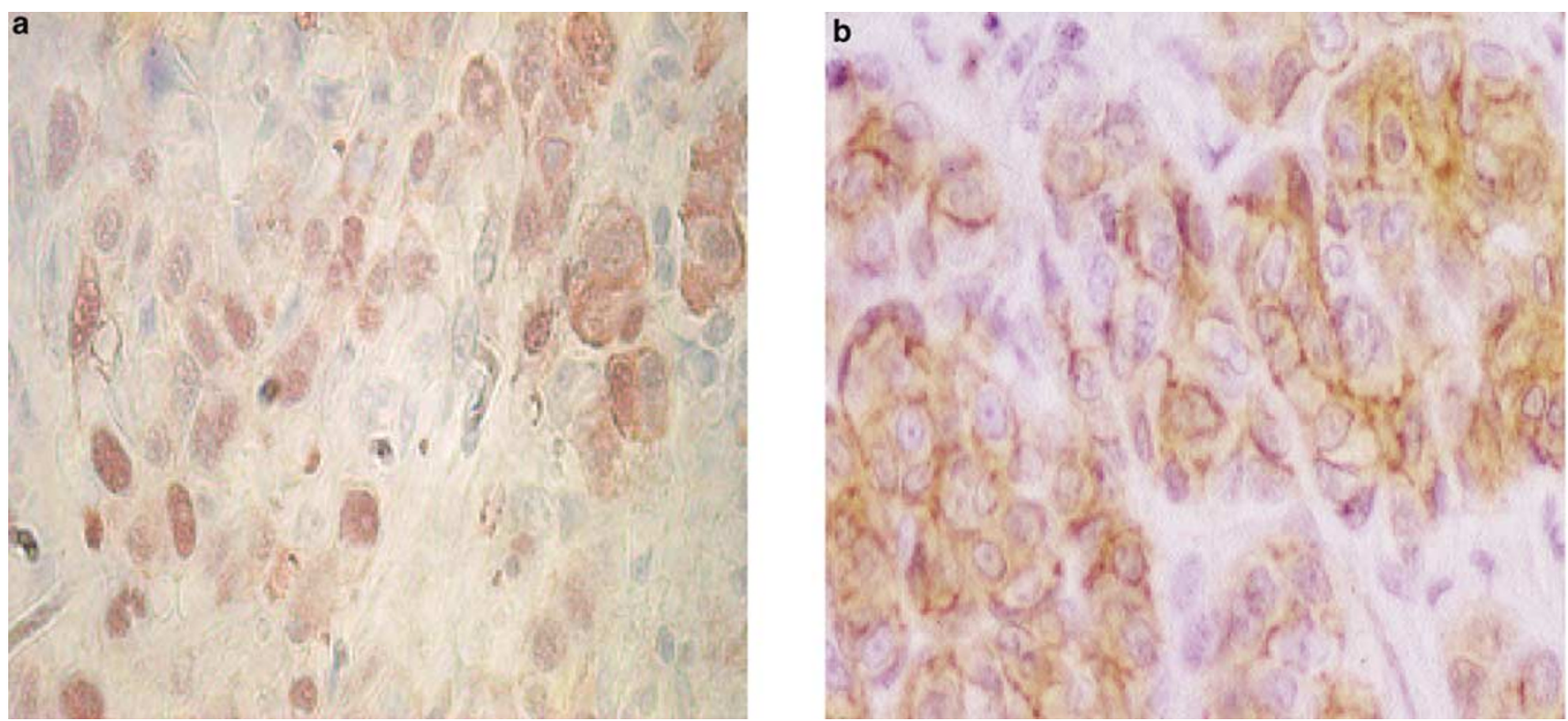

Figure 1 Immunohistochemical analysis showing primary superficial spreading melanomas with (a) cytoplasmic and nuclear staining of S100A4 and (b) membraneous and cytoplasmic staining of E-cadherin. 
Table 1 Number (precentage) of melanocytic tumors expressing (a) cytoplasmic and nuclear S100A4, and (b) membranous and cytoplasmic E-cadherin

(a) Cytoplasmic and nuclear S100A4

\begin{tabular}{|c|c|c|c|c|c|c|c|c|c|c|}
\hline \multirow[t]{2}{*}{ Type } & \multirow[t]{2}{*}{ Lesion } & \multirow[t]{2}{*}{ No. examined } & \multicolumn{4}{|c|}{ Cytoplasmatic expression ${ }^{\mathrm{a}}$} & \multicolumn{4}{|c|}{ Nuclear expression $^{\mathrm{a}}$} \\
\hline & & & 0 & $<5 \%$ & $5-50 \%$ & $>50 \%$ & 0 & $<5 \%$ & $5-50 \%$ & $>50 \%$ \\
\hline \multirow{2}{*}{ Superficial } & Primary & 99 & $56(57)$ & $5(5)$ & $24(24)$ & $14(14)$ & 77 (78) & $9(9)$ & $13(13)$ & 0 \\
\hline & Metastatic & 32 & $17(53)$ & $1(3)$ & $3(9)$ & $11(34)$ & 22 (69) & $4(13)$ & $6(19)$ & 0 \\
\hline \multirow{2}{*}{ Nodular } & Primary & 60 & $14(23)$ & $1(2)$ & $20(33)$ & $25(42)$ & $35(58)$ & $9(15)$ & $15(25)$ & $1(2)$ \\
\hline & Metastatic & 30 & $18(60)$ & $1(3)$ & $7(23)$ & $4(13)$ & $24(80)$ & $2(7)$ & $4(13)$ & \\
\hline Benign nevi & & 8 & $8(100)$ & 0 & 0 & 0 & $2(25)$ & $3(38)$ & $1(12)$ & $2(25)$ \\
\hline
\end{tabular}

(b) Membranous and cytoplasmic E-cadherin

\begin{tabular}{|c|c|c|c|c|c|c|c|c|c|c|}
\hline \multirow[t]{2}{*}{ Tyре } & \multirow[t]{2}{*}{ Lesion } & \multirow[t]{2}{*}{ No. examined } & \multicolumn{4}{|c|}{ Membranous expression ${ }^{\mathrm{a}}$} & \multicolumn{4}{|c|}{ Cytoplasmic expression ${ }^{\mathrm{a}}$} \\
\hline & & & 0 & $<5 \%$ & $5-50 \%$ & $>50 \%$ & 0 & $<5 \%$ & $5-50 \%$ & $>50 \%$ \\
\hline \multirow{2}{*}{ Superficial } & Primary & 92 & $10(11)$ & $34(37)$ & $38(41)$ & $10(11)$ & $12(13)$ & $8(9)$ & $13(14)$ & $59(64)$ \\
\hline & Metastatic & 26 & $10(38)$ & $5(19)$ & $9(35)$ & $2(8)$ & $13(50)$ & $1(4)$ & $3(11)$ & $9(35)$ \\
\hline \multirow{2}{*}{ Nodular } & Primary & 52 & $12(23)$ & $24(46)$ & $13(25)$ & $3(6)$ & $13(25)$ & $3(6)$ & $21(40)$ & $15(29)$ \\
\hline & Metastatic & 27 & $7(23)$ & $8(2)$ & $9(26)$ & $7(20)$ & $8(26)$ & $1(3)$ & $4(23)$ & $14(49)$ \\
\hline Benign nevi & & 8 & 0 & $2(25)$ & $6(75)$ & 0 & $1(12.5)$ & $2(25)$ & $4(50)$ & $1(12.5)$ \\
\hline
\end{tabular}

${ }^{\mathrm{a}}$ Scored as percentage of positively stained cells.

antibody recognizing the extracellular part of Ecadherin was used, and a significant correlation between membranous and cytoplasmic E-cadherin staining was observed. Taken together, this argued for considering membrane-associated and cytoplasmic E-cadherin as one parameter. Therefore, when performing the statistical analysis, cytoplasmic and membranous E-cadherin was combined in the same parameter. A high percentage of the examined cases demonstrated extended positive immunostaining and, the cutoff level between high and low expression of E-cadherin was set to $50 \%$. In contrast, a large fraction of the biopsies did not visualize any immunostaining for S100A4, and the cutoff level between high and low protein expression was in this case defined as positive vs negative staining.

When analyzing the whole panel, a significant correlation between the depth of primary lesion and S100A4 expression was detected $(P<0.001$, $N=155$ ), with thicker lesions expressing more S100A4. When the superficial spreading melanomas were analyzed separately, the same correlation was found $(P=0.02)$, while in the nodular subgroup, no significant relationships or clear tendencies were observed between positive immunostaining and tumor thickness (Table 2). In agreement with the observed correlation between tumor depth and expression of S100A4 when we examined the whole panel, longer disease-free survival $(P=0.054)$ was observed for patients having biopsies without S100A4 protein (Figure 2a). This tendency was also present in the superficial spreading subgroup $(P=0.074$ results not shown), but as expected, not for patients with nodular melanoma (result not shown). In order to look more closely into this relationship in the superficial spreading subgroup, we excluded all lesions thicker than $3.0 \mathrm{~mm}$ (Breslow category), and in the remaining group, consisting of 87 cases, the association between tumor thickness and S100A4 staining was even more pronounced $(P=0.008)$ (Table 2). Consistent with this, a significant increase in disease-free survival was observed for patients lacking S100A4 in their biopsies $(P=0.046$, Figure 2b). However, neither in this group did expression of the S100A4 protein in the biopsies significantly affect the overall survival of the patients (data not shown). When analyzing the panel of nodular melanomas in the same way, no 
Table 2 Relationship between expression of S100A4 or E-cadherin and mean depth of primary tumor growth

\begin{tabular}{|c|c|c|c|c|c|c|c|c|}
\hline Type of lesion & $\begin{array}{c}\text { Expression of } \\
\text { S100A4 }\end{array}$ & $\begin{array}{c}\text { Number } \\
\text { examined }\end{array}$ & $\begin{array}{c}\text { Depth of } \\
\text { growth (mm) }\end{array}$ & $\mathrm{P}$-value & $\begin{array}{c}\text { Expression of } \\
\text { E-cadherin }\end{array}$ & $\begin{array}{l}\text { Number } \\
\text { examined }\end{array}$ & $\begin{array}{c}\text { Depth of } \\
\text { growth (mm) }\end{array}$ & P-value \\
\hline \multirow{2}{*}{ All patients } & - & 68 & 2.23 & \multirow{2}{*}{0.0005} & $\leq 50 \%$ & 70 & 3.52 & \multirow[b]{2}{*}{0.002} \\
\hline & + & 87 & 3.36 & & $>50 \%$ & 71 & 2.24 & \\
\hline \multirow{2}{*}{ Nodular } & - & 14 & 4.89 & \multirow[b]{2}{*}{0.89} & $\leq 50 \%$ & 37 & 4.96 & \multirow{2}{*}{0.1} \\
\hline & + & 45 & 4.62 & & $>50 \%$ & 16 & 4.49 & \\
\hline \multirow{2}{*}{ Superficial } & - & 54 & 1.6 & \multirow{2}{*}{0.02} & $\leq 50 \%$ & 33 & 1.90 & \multirow{2}{*}{0.29} \\
\hline & + & 42 & 1.86 & & $>50 \%$ & 56 & 1.65 & \\
\hline \multirow[b]{2}{*}{ Superficial lesions $<3.0 \mathrm{~mm}$} & - & 49 & 1.07 & \multirow[b]{2}{*}{0.008} & $\leq 50 \%$ & 29 & 1.35 & \multirow[b]{2}{*}{0.256} \\
\hline & + & 33 & 1.42 & & $>50 \%$ & 51 & 1.11 & \\
\hline
\end{tabular}

a

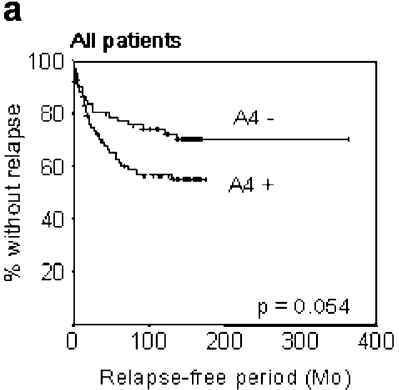

C

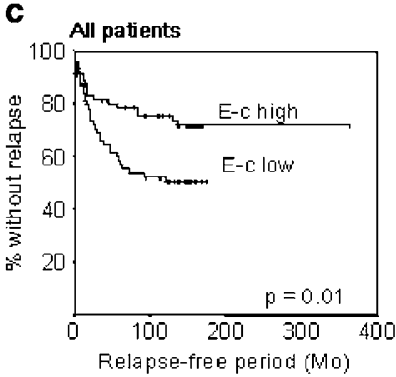

b

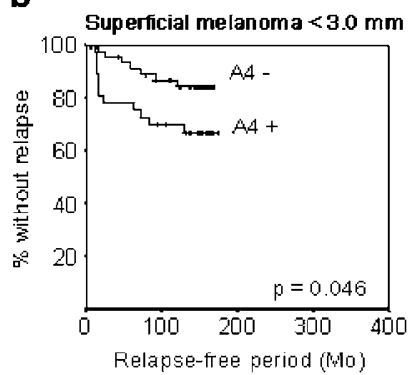

d

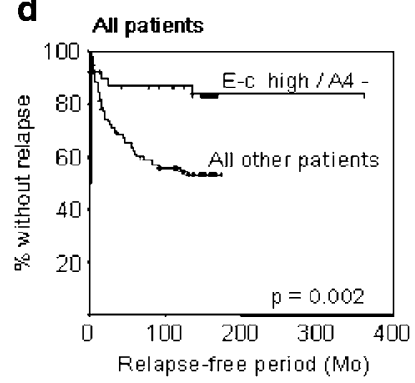

Figure 2 Kaplan-Meier curves demonstrating the relationship between disease-free survival and the expression of S100A4 (a, b), E-cadherin (c) or both proteins combined (d), in primary melanomas, for all the patients (a, c, d), or for only the superficial spreading melanomas thinner than $3.0 \mathrm{~mm}(\mathbf{b}) . \mathrm{E}-\mathrm{c}=\mathrm{E}$-cadherin and $\mathrm{A} 4=\mathrm{S} 100 \mathrm{~A} 4$.

significant relationship between tumor thickness/ disease-free survival and immunostaining of S100A4 was found in the remaining 24 cases.

When including all the biopsies in the analysis, a highly significant inverse relationship between primary tumor depth and expression of E-cadherin was revealed $(P=0.002)$ and furthermore, a significant decrease in disease-free survival was observed for patients having low E-cadherin levels $(P=0.01$, Figure 2c). We did not, however, observe the same

relationship when examining overall survival (results not shown), or when analyzing the melanoma subgroups (Table 2).

The results so far suggested the possibility of defining a new parameter, consisting of the combined expression of S100A4 and E-cadherin. The usefulness of this variable as a prognostic factor for malignant melanoma was examined in the whole panel of biopsies, as there were very few cases in the individual subgroups. Interestingly, patients having biopsies without S100A4 expression combined with high levels of E-cadherin expression had a significant longer disease-free survival $(P=0.002)$ with approximately $30 \%$ lower probability of relapse 15 years after time of diagnosis (Figure 2d), compared to the rest of the cases.

\section{Relationship between the Expression of S100A4, E-cadherin and Previously Analyzed Cell Cycle and Signal Transduction Factors}

This panel of malignant melanoma biopsies has previously been analyzed for expression of the proliferation marker Ki-67, in addition to various cell cycle factors (cyclin A, D1 and D3, p2 $1^{\mathrm{WAF} / \mathrm{CIPI}}$, p2 ${ }^{\text {Kip } 1}$ ), $\beta$-catenin and active ERK1/2. ${ }^{25-30}$ In the present study, the expression of S100A4 and Ecadherin was analyzed in relation to these factors. For S100A4, we found an inverse association with cytoplasmic $\beta$-catenin $(P=0.006)$, and a direct correlation to $\mathrm{p} 27^{\mathrm{Kip} 1}(P=0.01)$ and nuclear active MAP kinase $(P=0.012)$. Immunostaining of $\mathrm{E}$ cadherin correlated to immunostaining of $\beta$-catenin in the cytoplasm $(P<0.0001)$ and showed an inverse relationship with the proliferation marker Ki-67 $(P=0.004)$ and cyclin A (0.019). None of the other factors demonstrated significant associations to S100A4 or E-cadherin. 


\section{Discussion}

In the present study, S100A4 and E-cadherin protein expression was examined by immunohistochemistry in a panel of superficial spreading and nodular primary and metastatic melanomas, and evaluated their relation to clinical outcome. A significant larger fraction of nodular than superficial spreading primary melanomas expressed S100A4, a finding that is in agreement with the established conception of S100A4 as a metastasis-promoting protein., ${ }^{4,51}$ When examining the whole panel, as well as the superficial spreading melanoma subgroup, an association between the expression of S100A4 and the depth of the primary lesions was observed. This finding is in line with the observed tendency towards decreased disease-free survival for patients expressing S100A4 in their biopsies. In order to look more closely into this, we excluded, according to Breslow tumor thickness categories, ${ }^{32}$ all superficial spreading lesions thicker than $3.0 \mathrm{~mm}$ from the analysis. ${ }^{33}$ In the remaining group, the relationship between tumor thickness and S100A4 expression became more apparent, and again significantly shorter disease-free survival for patients with S100A4-positive biopsies was detected. Interestingly, even in the thinner Breslow category (lesions thinner than $1.5 \mathrm{~mm}$ ), the significant correlations between S100A4 and tumor depth/disease-free survival were sustained, suggesting that expression of S100A4 might be of particular importance for patient outcome in early-stage malignant melanoma. Our findings are in agreement with reports for breast ${ }^{13}$ and colorectal carcinoma, ${ }^{12}$ where S100A4 turned out to be the best prognostic factor in early stages of the disease. Interestingly, the association between S100A4 expression and vertical tumor thickness was not observed in the nodular melanomas, which in contrast to the superficial spreading melanomas have no radial growth phase. Altogether, these findings suggest that S100A4 exerts a function in the less advanced lesions making them more aggressive, and thus subsequently enhance the metastatic potential. Metastatic tumors from the nodular subgroup showed a tendency to lose S100A4 protein, both in the cytoplasm and in the nucleus. This tendency was not visible in metastatic tumors from the superficial spreading subgroup, again implicating a different role for S100A4 in progression of the two subgroups of malignant melanoma. The expression of S100A4 in metastatic lesions did not affect patient survival, a finding that agrees well with a previous study from our group, examining the S100A4 mRNA level in 45 distant metastases from malignant melanomas. ${ }^{34}$

The loss of E-cadherin expression has been associated with malignant progression through loss of cell-cell contact and thereby allowing cancer cells to detach and migrate. ${ }^{35,36}$ Several studies have described E-cadherin expression in the cytoplasm. ${ }^{37}$ Le et $a l^{24,38}$ recently reported that E-cadherin located on the cell surface was constitutively internalized and recycled back to the surface in a PKC-dependent manner. The anti-E-cadherin-antibody used in this study is well characterized, and has been shown to bind the extracellular $\mathrm{Ca}^{2+}$-binding domains that are responsible for establishing the cell-cell contact. $^{39}$ We therefore hypothesize that E-cadherin observed in the cytoplasm was fully functional and able to participate in cell-cell adhesion when recycled back to the cell surface. For this reason, E-cadherin staining at the plasma membrane and in the cytosol was combined in one parameter when performing the statistical analysis.

When all the primary lesions were analyzed, the expression of E-cadherin was significantly correlated to the depth of the primary tumor. Accordingly, disease-free survival for patients with low E-cadherin expression was significantly reduced. This association was, however, not observed when the melanoma subgroups were analyzed individually. Multiple studies have demonstrated that loss of E-cadherin expression correlates with tumor progression. ${ }^{20,40}$ However, in contrast to the metastasis suppressor role often described for E-cadherin, Silye et al, ${ }^{41}$ reported loss of E-cadherin in dermal nests of melanomas in radial growth phase, and expression in a high proportion of melanomas in their vertical growth phase, suggesting that in melanomas Ecadherin expression is modulated during invasion and metastasis. In our study, an inverse correlation between S100A4 and E-cadherin was observed, with E-cadherin being significantly higher expressed in primary superficial spreading lesions compared to in nodular melanomas. This is in agreement with several previous studies reporting inverse expression of these two factors. ${ }^{14,16,42}$ Our study demonstrates, in line with reports on non-small-cell lung- and gastric-cancer patients, ${ }^{14,15}$ that high Ecadherin expression combined with negative S100A4 status, predicts longer disease-free survival for melanoma patients.

The relationship between expression of S100A4, E-cadherin and previously analyzed cell cycle and signal transduction proteins was also examined. Surprisingly, expression of S100A4 correlated to the expression of the cell cycle inhibitor $\mathrm{p} 27^{\mathrm{Kip} 1}$. This is in contrast to several studies reporting that loss of p2 $7^{\text {Kip } 1}$ expression is associated with lower patient survival. ${ }^{20,27,43}$ Our finding agrees, however, with those of a recent study communicating a strong correlation between $\mathrm{p} 27^{\mathrm{Kip} 1}$ and the presence of axillary lymph node metastasis in breast cancer patients, ${ }^{44}$ suggesting that other mechanisms, such as accumulation of cytoplasmic p27 $7^{\mathrm{Kip} 145}$ or $\mathrm{p} 27^{\mathrm{Kip} 1}$ mediated protection from apoptosis ${ }^{46}$ may mask the cell cycle-inhibiting effect of the protein. Furthermore, constitutive ERK1/2 activation or oncogenic Ras has been shown to lead to deregulation of or cytoplasmic sequestering of p27 Kip1 ${ }^{47,48}$ Intriguingly, a correlation between activated nuclear ERK1/2 and S100A4 was revealed. This observation, combined 
with previous data showing correlation between p2 $7^{\text {Kip } 1}$ and activated nuclear ERK $1 / 2,{ }^{30}$ makes it natural to speculate whether these three factors interplay in the process leading to a metastatic phenotype. Although it has been reported that p2 $7^{\text {Kip1 }}$ might in part be regulated by E-cadherin, we did not find any significant correlation between the expressions of the two proteins. ${ }^{49}$ In conclusion, our study adds malignant melanoma to the list of cancers where S100A4 contributes to an aggressive phenotype, and the protein might be particularly important in early superficial spreading melanomas. The expression of S100A4 and E-cadherin in malignant melanoma biopsies was inversely correlated and both markers might be used as predictors of disease-free survival. When the two factors were combined, a new variable with more significant prediction of disease progression was revealed. The fact that approximately one-third of the patients diagnosed with metastatic melanoma present without regional lymph node involvement, and the lack of effective systemic therapies, ${ }^{50}$ makes it of great importance to identify patients at increased risk for metastatic disease. The present results suggest that expression levels of S100A4 and/or E-cadherin might be prognostic parameters for patients with nonmetastatic melanoma, and call for further studies to clarify the potential that these two proteins have as prognostic markers in early melanoma development.

\section{Acknowledgements}

We are grateful to Ellen Hellesylt, Mette Ingrud and Liv Inger Hårseth for excellent technical assistance. This work was supported by the Norwegian Research Council and The Norwegian Cancer Society.

\section{References}

1 Hauschild A, Engel G, Brenner W, et al. Predictive value of serum S100B for monitoring patients with metastatic melanoma during chemotherapy and/or immunotherapy. Br J Dermatol 1999;140:1065-1071.

2 Banfalvi T, Udvarhelyi N, Orosz Z, et al. Heterogenous $\mathrm{S}-100 \mathrm{~B}$ protein expression patterns in malignant melanoma and association with serum protein levels. Oncology 2003;64:374-379.

3 Donato R. Intracellular and Extracellular Roles of S100 Proteins. Microsc. Res. Tech. 2003;60:540-551.

4 Maelandsmo GM, Hovig E, Skrede M, et al. Reversal of the in vivo metastatic phenotype of human tumor cells by an anti-CAPL (mts1) ribozyme. Cancer Res 1996;56:5490-5498.

5 Takenaga K, Nakamura Y, Sakiyama S. Expression of antisense RNA to S100A4 gene encoding an S100related calcium-binding protein suppresses metastatic potential of high-metastatic Lewis lung carcinoma cells. Oncogene 1997;14:331-337.
6 Bjornland K, Winberg JO, Odegaard OT, et al. S100A4 involvement in metastasis: deregulation of matrix metalloproteinases and tissue inhibitors of matrix metalloproteinases in osteosarcoma cells transfected with an anti-S100A4 ribozyme. Cancer Res 1999;59: 4702-4708.

7 Kriajevska M, Tarabykina S, Bronstein I, et al. Metastasis-associated Mts1 (S100A4) protein modulates protein kinase $\mathrm{C}$ phosphorylation of the heavy chain of nonmuscle myosin. J Biol Chem 1998;273: 9852-9856.

8 Kriajevska M, Bronstein IB, Scott DJ, et al. Metastasisassociated protein Mts1 (S100A4) inhibits CK2mediated phosphorylation and self-assembly of the heavy chain of nonmuscle myosin. Biochim Biophys Acta 2000;1498:252-263.

9 Ambartsumian N, Klingelhofer J, Grigorian M, et al. The metastasis-associated Mts1(S100A4) protein could act as an angiogenic factor. Oncogene 2001;20: 4685-4695.

10 Nakamura T, Ajiki T, Murao S, et al. Prognostic significance of S100A4 expression in gallbladder cancer. Int J Oncol 2002;20:937-941.

11 Ninomiya I, Ohta T, Fushida S, et al. Increased expression of S100A4 and its prognostic significance in esophageal squamous cell carcinoma. Int J Oncol 2001;18:715-720.

12 Gongoll S, Peters G, Mengel M, et al. Prognostic significance of calcium-binding protein S100A4 in colorectal cancer. Gastroenterology 2002;123: 1478-1484.

13 Rudland PS, Platt-Higgins A, Renshaw C, et al. Prognostic significance of the metastasis-inducing protein S100A4 (p9Ka) in human breast cancer. Cancer Res 2000;60:1595-1603.

14 Yonemura Y, Endou Y, Kimura K, et al. Inverse expression of S100A4 and E-cadherin is associated with metastatic potential in gastric cancer. Clin Cancer Res 2000;6:4234-4242.

15 Kimura K, Endo Y, Yonemura Y, et al. Clinical significance of S100A4 and E-cadherin-related adhesion molecules in non-small cell lung cancer. Int J Oncol 2000;16:1125-1131.

16 Kohya N, Kitajima Y, Jiao W, et al. Effects of E-cadherin transfection on gene expression of a gallbladder carcinoma cell line: repression of MTS1/S100A4 gene expression. Int J Cancer 2003;104:44-53.

17 Takeichi M. Cadherin cell adhesion receptors as a morphogenetic regulator. Science 1991;251:1451-1455.

18 Wheelock MJ, Jensen PJ. Regulation of keratinocyte intercellular junction organization and epidermal morphogenesis by E-cadherin. J Cell Biol 1992;117: 415-425.

19 Tang A, Eller MS, Hara M, et al. E-cadherin is the major mediator of human melanocyte adhesion to keratinocytes in vitro. J Cell Sci 1994;107:983-992.

20 Johnson JP. Cell adhesion molecules in the development and progression of malignant melanoma. Cancer Metastasis Rev 1999;18:345-357.

21 Heimann R, Lan F, McBride R, et al. Separating favorable from unfavorable prognostic markers in breast cancer: the role of E-cadherin. Cancer Res 2000;60:298-304.

22 Flatmark K, Pedersen KB, Nesland JM, et al. Nuclear localization of the metastasis related protein S100A4 correlates with tumor stage in colorectal cancer. J Pathol 2003;200:589-595. 
23 Pedrocchi M, Schafer BW, Durussel I, et al. Purification and characterization of the recombinant human calcium-binding S100 proteins CAPL and CACY. Biochemistry 1994;33:6732-6738.

24 Le TL, Yap AS, Stow JL. Recycling of E-cadherin: a potential mechanism for regulating cadherin dynamics. J Cell Biol 1999;219-232.

25 Florenes VA, Maelandsmo GM, Faye R, et al. Cyclin A expression in superficial spreading malignant melanomas correlates with clinical outcome. J Pathol 2001; 195:530-536.

26 Florenes VA, Faye RS, Maelandsmo GM, et al. Levels of cyclin D1 and D3 in malignant melanoma: deregulated cyclin D3 expression is associated with poor clinical outcome in superficial melanoma. Clin Cancer Res 2000;6:3614-3620.

27 Florenes VA, Maelandsmo GM, Kerbel RS, et al. Protein expression of the cell-cycle inhibitor p27Kip1 in malignant melanoma: inverse correlation with disease-free survival. Am J Pathol 1998;153:305-312.

28 Maelandsmo GM, Holm R, Fodstad O, et al. Cyclin kinase inhibitor p21WAF1/CIP1 in malignant melanoma: reduced expression in metastatic lesions. Am J Pathol 1996;149:1813-1822.

29 Maelandsmo GM, Holm R, Flørenes VA. Reduced betacatenin Expression in the Cytoplasm of Advanced Stage Superficial Spreading melanoma. Clin Cancer Res 2003;19:3383-3388.

30 Jørgensen K, Holm R, Mælandsmo GM, et al. Expression of activated ERK1/2 in malignant melanomas; Relationship to clinical outcome. Clin Cancer Res 2003;9:5325-5331.

31 Levett D, Flecknell PA, Rudland PS, et al. Transfection of S100A4 produces metastatic variants of an orthotopic model of bladder cancer. Am J Pathol 2002; 160:693-700.

32 Owen SA, Sanders LL, Edwards LJ, et al. Identification of higher risk thin melanomas should be based on Breslow depth not Clark level IV. Cancer 2001;91: 983-991.

33 Breslow A. Thickness, cross-sectional areas and depth of invasion in the prognosis of cutaneous melanoma. Ann Surg 1970;172:902-908.

34 Maelandsmo GM, Florenes VA, Mellingsaeter T, et al. Differential expression patterns of S100A2, S100A4 and S100A6 during progression of human malignant melanoma. Int J Cancer 1997;74:464-469.

35 Hirohashi S. Inactivation of the E-cadherin-mediated cell adhesion system in human cancers. Am J Pathol 1998;153:333-339.

36 Behrens J. Cadherins and catenins: role in signal transduction and tumor progression. Cancer Metastasis Rev 1999;18:15-30.

37 Paterson AD, Parton RG, Ferguson C, et al. Characterization of E-cadherin endocytosis in isolated MCF-7 and chinese hamster ovary cells: the initial fate of unbound E-cadherin. J Biol Chem 2003;278:2105021057.

38 Le TL, Joseph SR, Yap AS, et al. Protein kinase C regulates endocytosis and recycling of E-cadherin. Am J Physiol Cell Physiol 2002;283:489-499.

39 Symons JR, LeVea CM, Mooney RA. Expression of the leucocyte common antigen-related (LAR) tyrosine phosphatase is regulated by cell density through functional E-cadherin complexes. Biochem J 2002; 365:513-519.

40 Pignatelli M, Ansari TW, Gunter P, et al. Loss of membranous E-cadherin expression in pancreatic cancer: correlation with lymph node metastasis, high grade, and advanced stage. J Pathol 1994;174:243-248.

41 Silye R, Karayiannakis AJ, Syrigos KN, et al. Ecadherin/catenin complex in benign and malignant melanocytic lesions. J Pathol 1998;186:350-355.

42 Keirsebilck A, Bonne S, Bruyneel E, et al. E-cadherin and metastasin (mts-1/S100A4) expression levels are inversely regulated in two tumor cell families. Cancer Res 1998;58:4587-4591.

43 Kirla RM, Haapasalo HK, Kalimo $\mathrm{H}$, et al. Low expression of p27 indicates a poor prognosis in patients with high-grade astrocytomas. Cancer 2003; 97:644-648.

44 Kouvaraki M, Gorgoulis VG, Rassidakis GZ, et al. High expression levels of p27 correlate with lymph node status in a subset of advanced invasive breast carcinomas: relation to E-cadherin alterations, proliferative activity, and ploidy of the tumors. Cancer 2002;94: 2454-2465.

45 Baldassarre G, Belletti B, Bruni P, et al. Overexpressed cyclin D3 contributes to retaining the growth inhibitor p27 in the cytoplasm of thyroid tumor cells. J Clin Invest 1999;104:865-874.

46 Kolar Z, Flavell JR, Ehrmann Jr J, et al. Apoptosis of malignant cells in Hodgkin's disease is related to expression of the cdk inhibitor p27KIP1. J Pathol 2000; 190:604-612.

47 Liu X, Sun Y, Ehrlich M, et al. Disruption of TGFbeta growth inhibition by oncogenic ras is linked to p27Kip1 mislocalization. Oncogene 2000;19: 5926-5935.

48 Cheng M, Sexl V, Sherr CJ, et al. Assembly of cyclin Ddependent kinase and titration of p27Kip1 regulated by mitogen-activated protein kinase kinase (MEK1). Proc Natl Acad Sci USA 1998;95:1091-1096.

49 St Croix B, Sheehan C, Rak JW, et al. E-Cadherindependent growth suppression is mediated by the cyclin-dependent kinase inhibitor p27(KIP1). J Cell Biol 1998;142:557-571.

50 Carlson JA, Slominski A, Linette GP, et al. Biomarkers in melanoma: predisposition, screening and diagnosis. Expert Rev Mol Diagn 2003;2:163-184. 